http://jmscr.igmpublication.org/home/ ISSN (e)-2347-176x ISSN (p) 2455-0450 crossref DOI: https://dx.doi.org/10.18535/jmscr/v8i1.22

\title{
Histopathological evaluation of bone lesions in tertiary care centre
}

\author{
Authors \\ Dr M A Sameer, Dr Rupali G Sonwane* \\ Dr Shankarrao Chavan Government Medical College Vishnupuri, Nanded \\ *Corresponding Author \\ Dr Rupali G Sonwane
}

\begin{abstract}
Objectives: To study the histopathological features of bone lesion and correlate them with age, site and type of lesions.

Material and Methods: This study was undertaken in department of pathology, Dr SCGMC, Nanded for period of 3 years from November 2016-November 2019to determine the histopathological findings in bone lesions.

Results: Total 60 cases were studied out which nonneoplastic lesions comprised 32 cases (53.33\%), 21 cases (35\%) had benign tumors and malignant tumors accounted for 7 cases (11.66\%). Bone lesions were more common in $<20$ years age group (31.66\%). Male to female ratio in bone lesions is 1.5:1. The most common site of bone lesion is femur (20/60), followed by tibia (15/60), humerus (11/60), radius (7/60), ulna (4/60), small bones (3/60).

Conclusion: The most common lesions are non neoplastic followed by benign neoplastic tumors then malignant neoplastic tumors. All are more common in younger age group with male predominance. The most common site involved is femur.

Keywords: Non neoplastic, neoplastic, bone lesions.
\end{abstract}

\section{Introduction}

Bone is made of cartilage, osteoid, fibrous tissue, and bone marrow elements, with each tissue having a potential to develop a lesion either benign or malignant ${ }^{1}$. The gamut of bone lesions ranges from inflammation, degenerative changes, and metabolic diseases to neoplasm ${ }^{2,3}$. A spectrum of pathological bone lesions can be presented in any form from inflammatory to neoplastic conditions. It is important to remember, that some benign processes such as osteomyelitis can mimic malignant tumours, and some malignant lesions like metastases or myeloma, can mimic benign. It is difficult to determine radiologically with plain film imaging whether a bone lesion is benign or malignant ${ }^{4}$. Bone tumours and tumour-like lesions occur mainly between the first and fourth decades of life. It is therefore clear that these tumours have a potentially devastating effect on the most productive segment of the population ${ }^{5}$. Neoplasms and tumour like conditions of bone are rare. Thus, orthopaedic surgeons, radiologists, and pathologists generally have little experience with these lesions. Bone lesions often pose diagnostic challenges to surgical pathologists. Therefore, an integrated approach involving radiographic, 
histologic, and clinical data are necessary to form an accurate diagnosis and to determine the degree of activity and malignancy of each lesion ${ }^{6}$. Definitive clinical diagnosis of bone lesion is often difficult, so it is essential to identify the lesion correctly before deciding the line of treatment, be it simple currettement, excision surgery, amputation or irradiation ${ }^{7}$.

\section{Aims and Objective}

To study the histopathological features of bone lesion and correlate them with age, site and type of lesions.

\section{Material and Methods}

This study conducted in the Department of Pathology at SCGMC, Vishnupuri, Nanded over a period of 3 years from November 2016-November 2019. All the histopathological reports and slides of patients who had bone tissue biopsies were reviewed to provide relevant information on age, sex, histopathological interpretation, and the anatomical site of occurrence. All tumours of hematopoietic and odontogenic origin were excluded in this study. Bony along with soft tissue biopsy or in some cases amputated limb was received, and thorough gross examination of each lesion was done. Soft tissue of each biopsy was immediately fixed into $10 \%$ formalin and processed by paraffin embedding. Bone from each biopsy was kept for decalcification in $10 \%$ nitric acid. After that, fixation in $10 \%$ formalin, processing, section cutting and haematoxylin and eosin staining was performed.

\section{Results}

A histopathological study of various bone lesions was carried out at a teaching hospital from November 2016-November 2019. During these 3 years, total 60 cases were studied. Nonneoplastic lesions comprised 32 cases $(53.33 \%), 21$ cases $(35 \%)$ had benign tumors and malignant tumors accounted for 7 cases (11.66\%).
Table No 1: Proportion of different bone lesions

\begin{tabular}{|l|c|c|}
\hline \multirow{2}{*}{ Non Neoplastic } & \multicolumn{2}{|c|}{ Neoplastic } \\
\cline { 2 - 3 } & Benign & Malignant \\
\hline Osteomylitis- 16 & Osteochondroma-8 & Mets -2 \\
\hline $\begin{array}{l}\text { TB } \\
\text { osteomyelitis -8 }\end{array}$ & Osteoid osteoma- 4 & Osteosarcoma -2 \\
\hline $\begin{array}{l}\text { Aneurismal } \\
\text { bone cyst- 5 }\end{array}$ & Chondroblastoma- 2 & Ewing sarcoma- \\
\hline $\begin{array}{l}\text { Nonossifying } \\
\text { fibroma-1 }\end{array}$ & Gaint cell tumor- 7 & Chordoma-1 \\
\hline $\begin{array}{l}\text { Simple bone } \\
\text { cyst-2 }\end{array}$ & & \\
\hline Total-32 & Total- 21 & Total- 7 \\
\hline
\end{tabular}

Amongst non-neoplastic lesions, chronic osteomyelitis (17 cases, $53.13 \%$ ) were commonest followed by tuberculous Osteomyelitis (8 cases, $25 \%$ ) and aneurysmal bone cyst (5 cases, 15.62 $\%$ ) while Osteochondromas (8 cases, $38.1 \%$ ) was more common in the category of benign neoplastic lesions followed by giant cell tumour (7 cases, 33.33\%). Osteoma (4 cases, 19\%) and chondroblastoma (2 cases, 9.52\%) were common neoplastic bone lesions. Among malignant tumours, sarcoma accounts for 4 cases (osteosarcoma 2, and Ewing's sarcoma 2) followed by metastatic carcinoma (2 cases), chordoma (1 cases).

Table No 2: Age wise distribution of bone lesions

\begin{tabular}{|l|c|c|c|c|}
\hline \multirow{2}{*}{$\begin{array}{l}\text { Age } \\
\text { (years) }\end{array}$} & \multirow{2}{*}{$\begin{array}{c}\text { Non } \\
\text { neoplastic }\end{array}$} & \multicolumn{2}{|c|}{ Neoplastic } & Total \\
\cline { 3 - 4 } & 10 & 8 & 1 & \\
\hline$<20$ years & 9 & 5 & 3 & 19 \\
\hline $21-40$ & 8 & 7 & 1 & 16 \\
\hline $41-60$ & 5 & 1 & 2 & 8 \\
\hline$>60$ & & &
\end{tabular}

Bone lesions were more common in $<20$ years age group (31.66\%). 
Graph No 1: Age wise distribution of bone lesions

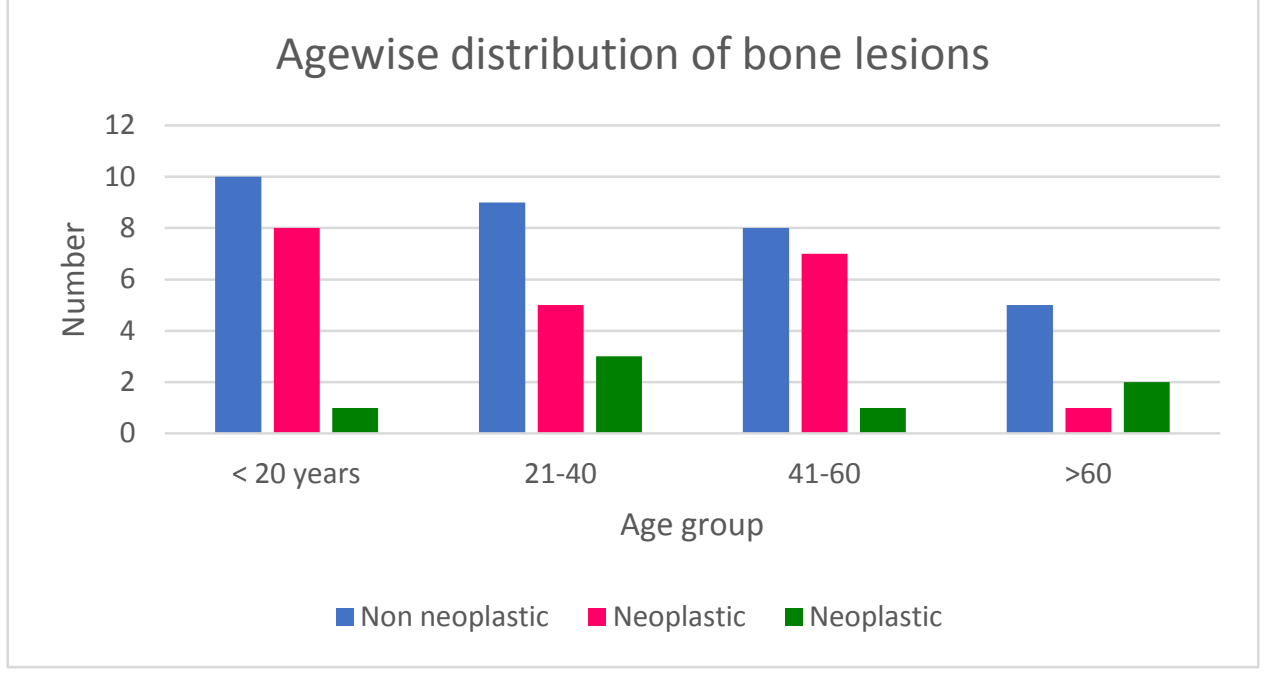

Table No 3: Sex distribution of bone lesion

\begin{tabular}{|l|c|c|c|c|}
\hline \multirow{2}{*}{ Bone lesions } & \multirow{2}{*}{ Non neoplastic } & \multicolumn{2}{|c|}{ Neoplastic } & Total \\
\cline { 3 - 4 } & & Benign & Malignant & \\
\hline Male & 19 & 12 & 5 & 36 \\
\hline Female & 13 & 9 & 2 & 24 \\
\hline Total & 32 & 21 & 7 & 60 \\
\hline
\end{tabular}

Males (36 out of 60 cases, $60 \%$ ) were affected more commonly than females ( 24 out of 60 cases, $40 \%$ ) with male to female ratio as $1.5: 1$.

Graph No 2: Sex distribution of bone lesion

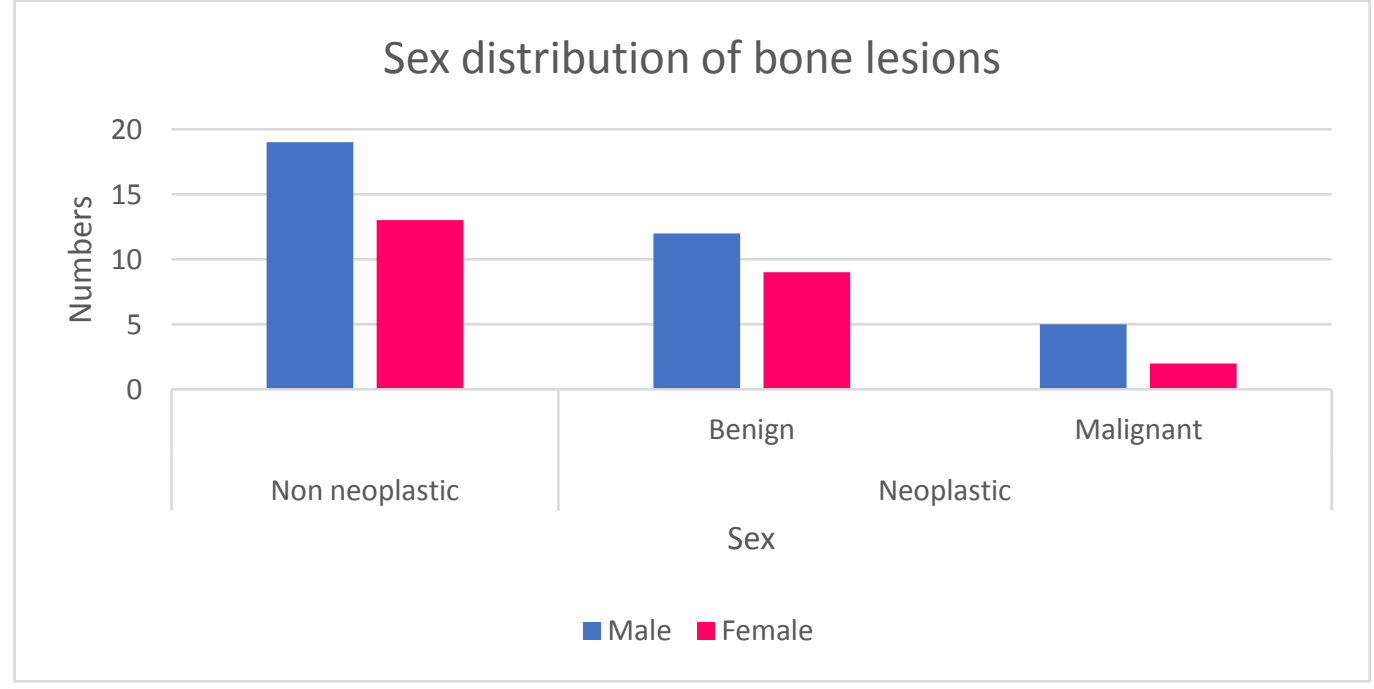

Table No 4: Site distribution of bone lesions

\begin{tabular}{|l|c|c|c|}
\hline Location & Non neoplastic & Neoplastic & Total \\
\hline Femur & 11 & 9 & 20 \\
\hline Tibia & 8 & 7 & 15 \\
\hline Humerus & 6 & 5 & 11 \\
\hline Radius & 4 & 3 & 7 \\
\hline Ulna & 2 & 2 & 4 \\
\hline Small bones & 1 & 2 & 3 \\
\hline Total & 32 & 28 & 60 \\
\hline
\end{tabular}

The most common site of bone lesion is femur (11/60), radius (7/60), ulna (4/60), small bones (20/60), followed by tibia (15/60), humerus (3/60). 
Graph No 3: Site distribution of bone lesions

\section{Site distribution of bone lesions}

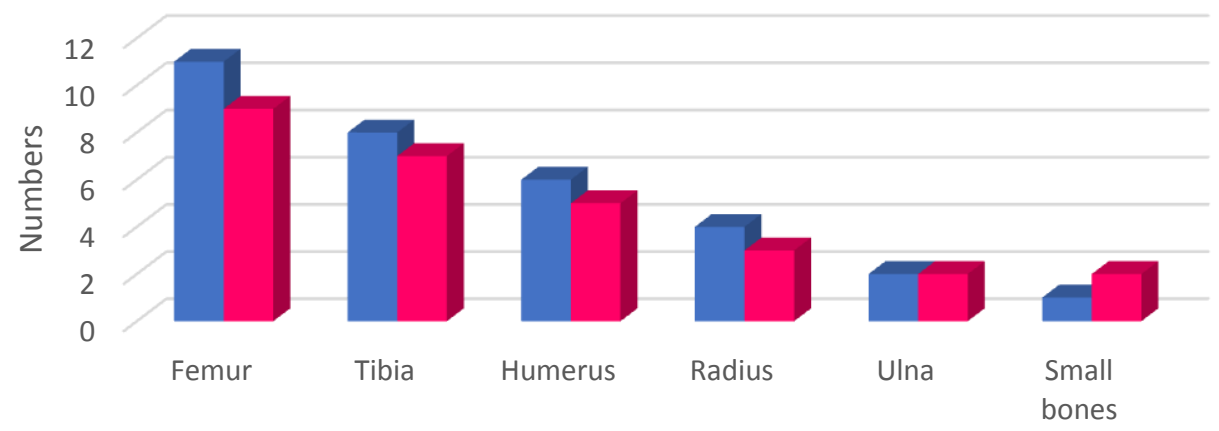

site

\section{Non neoplastic $\square$ Neoplastic}

\section{Osteomyelitis:}

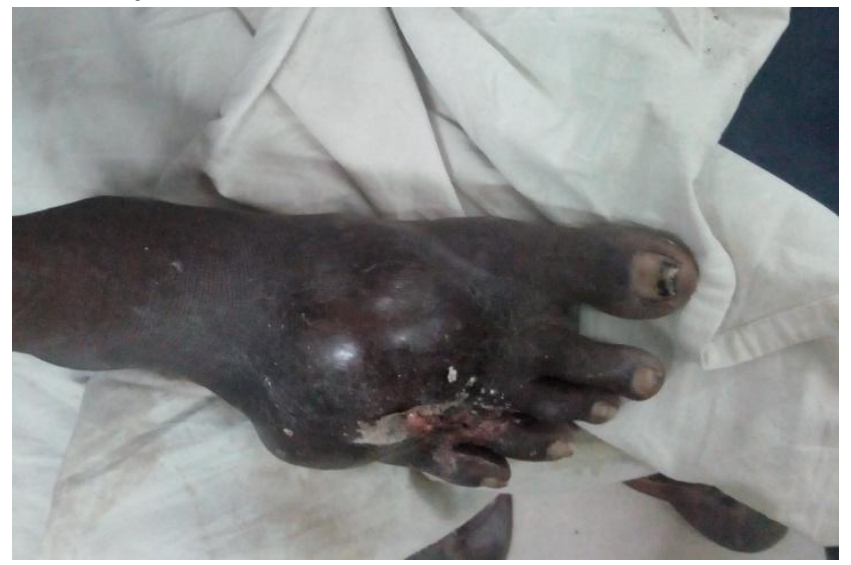

Fig No 1(A) (Osteomyelitis). Gross photograph of osteomyelitis of right foot.

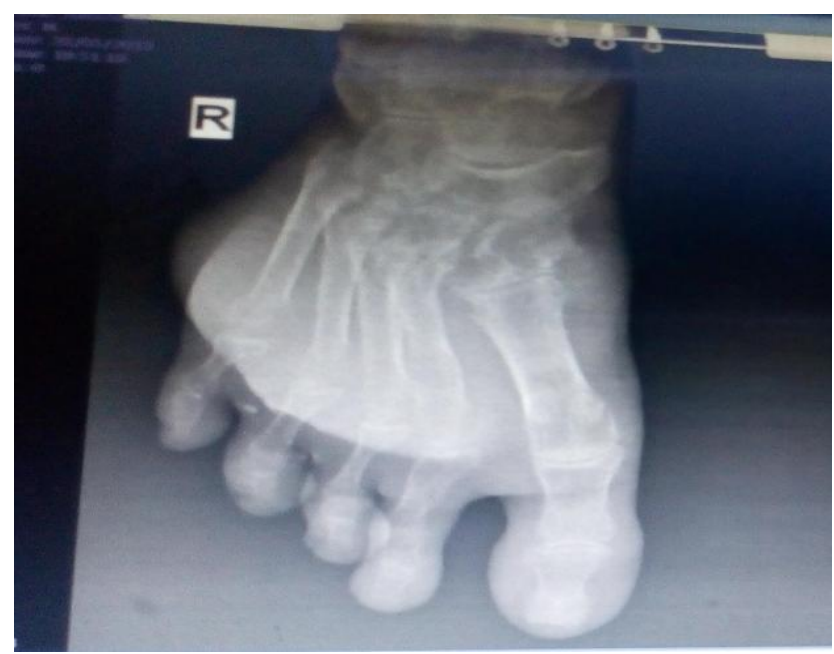

Fig No 1 (B) (Osteomyelitis). $\mathrm{X}$ ray of osteomyelitis of right foot.

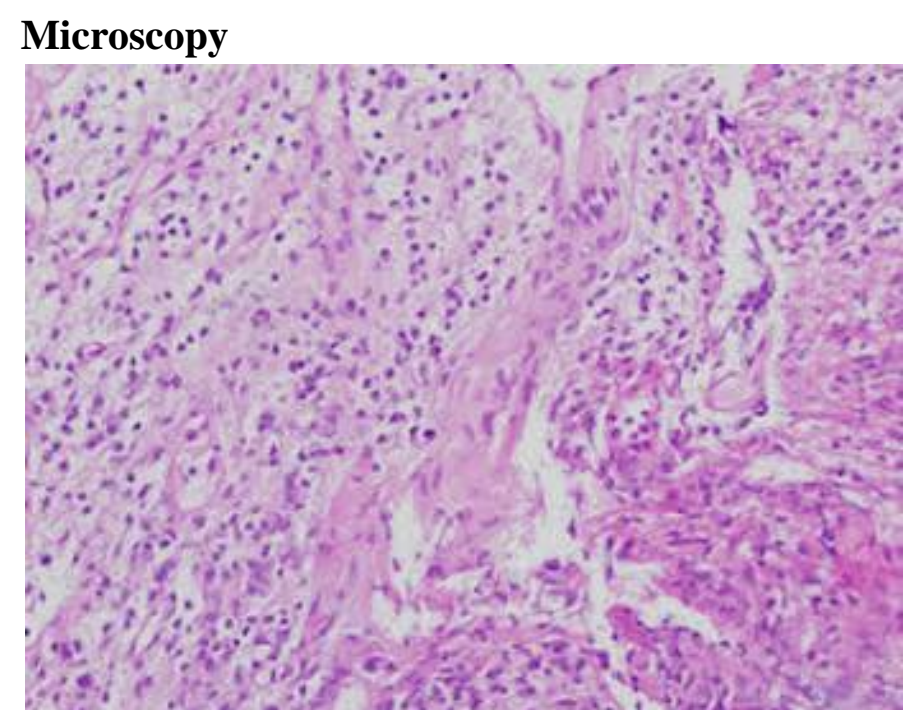

Fig No 1 (C) (Osteomyelitis) Section study shows inflammatory infiltrate comprised of lymphocytes, plasma cells along with fibrosis at places.

\section{Osteosarcoma}

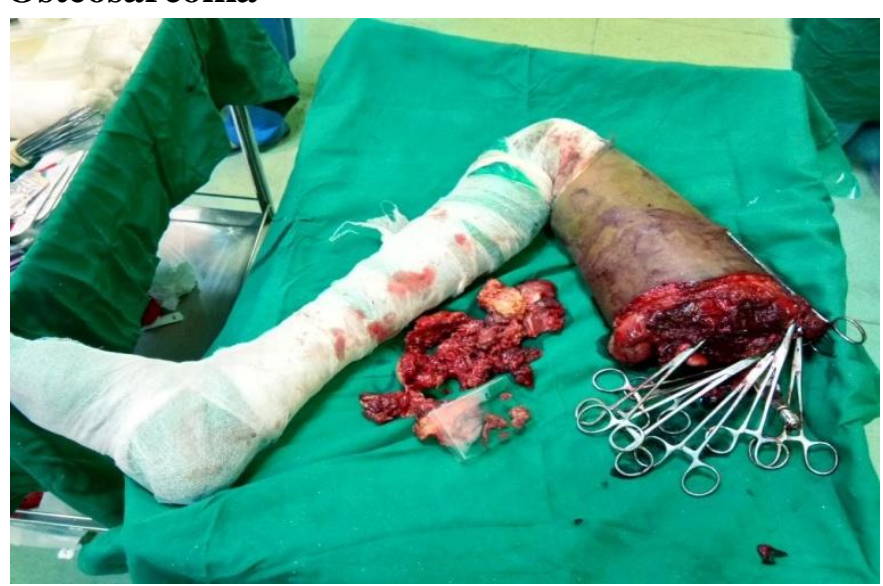

Fig No 2 (A) (Osteosarcoma). postoperative photograph high thigh amputation for osteosarcoma of proximal right femur. 


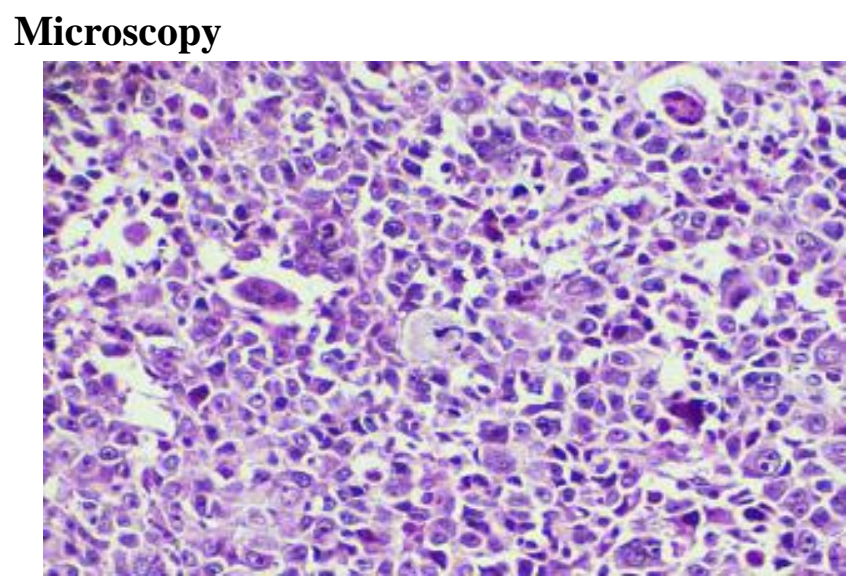

Fig No 2 (B) (Osteosarcoma). Section study shows atypical neoplastic hyperchromatic osteoblast along with giant cells and fibroblast.

\section{Chondroblastoma}

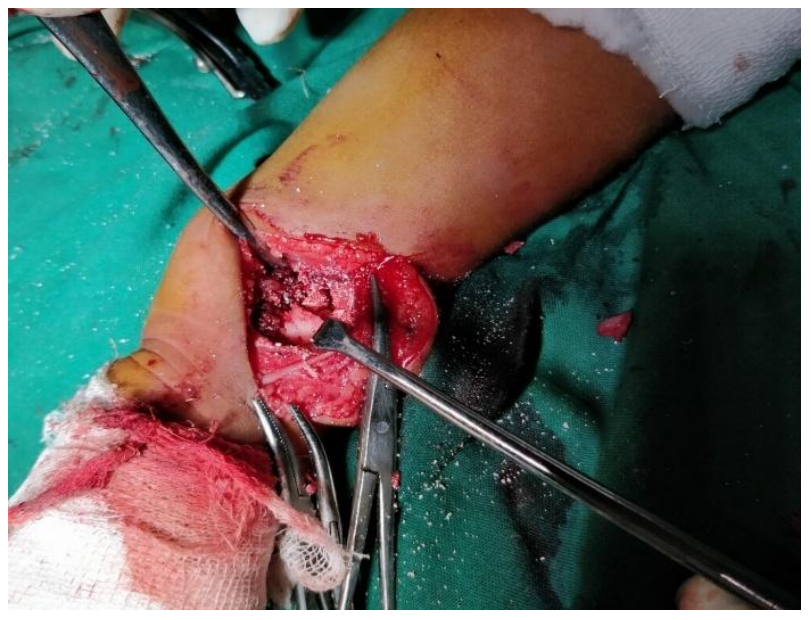

Fig No 3(A) (Chondroblastoma): intraoperative photograph of chondroblastoma of right distal radius.

\section{Microscopy}

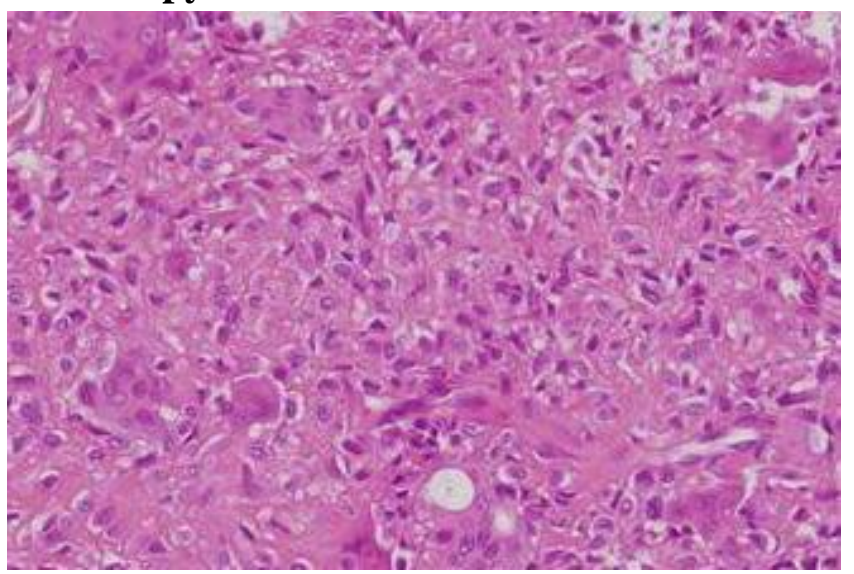

Fig No3 (B) (Chondroblastoma) . Section study shows polyhedral chondroblasts with abundant pink cytoplasm and variable pigment, well defined cell borders and hyperlobulated nuclei with grooves in mineralized, chicken wire matrix that surrounds chondroblasts along with giant cells.

\section{Osteochondroma}

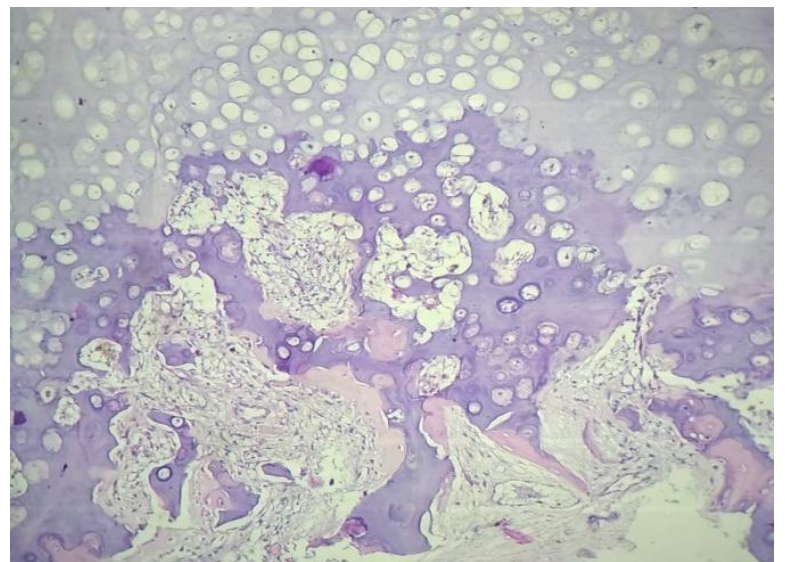

Fig No 4(B) (Osteochondroma) Section study shows mature hyaline cartilage with overlying fibrous perichondrium, transition between bone and cartilage cap resembles growth plate, showing endochondral ossification into mature bone.

\section{Non ossifying fibroma}

Microscopy

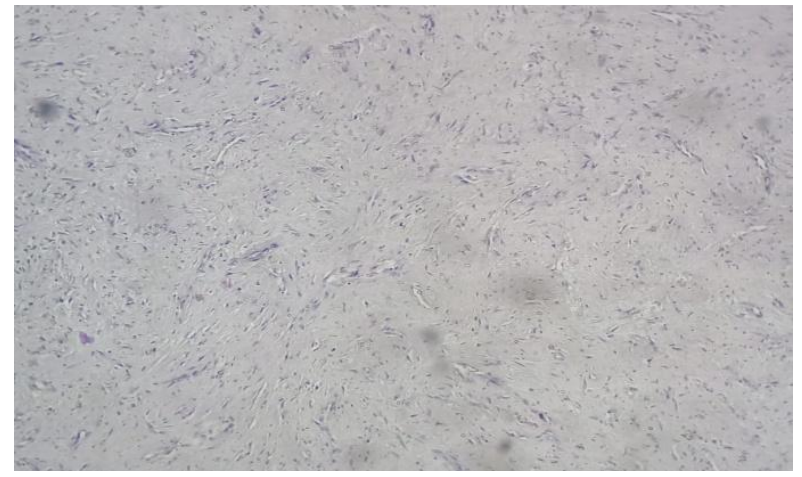

Fig No. 5 (Non ossifying fibroma). Section study shows fibroblastic spindle cells in whirled or storiform pattern, fibroblastic connective tissue background

\section{Ewing sarcoma}

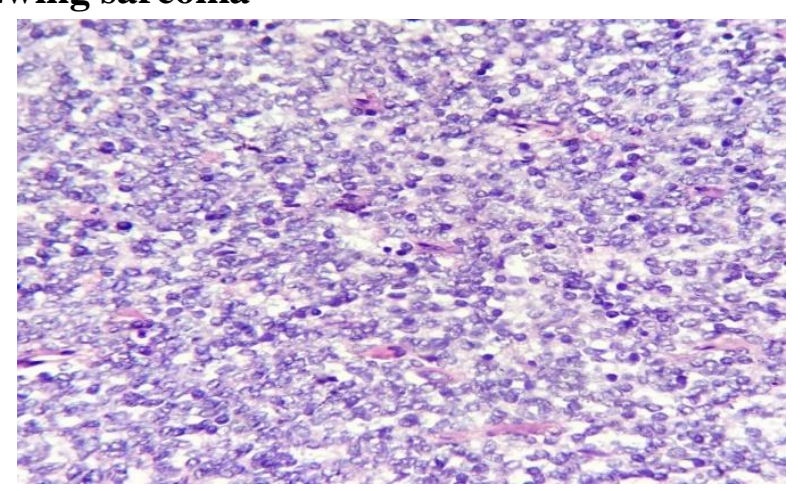

Fig No 6. (Ewing sarcoma). Section study shows sheets of small, round, uniform cells having round nuclei with indentations, small nucleoli with indistinct cell membranes and scant cytoplasm. At places few mitoses seen. 


\section{Tuberculous osteomyelitis}

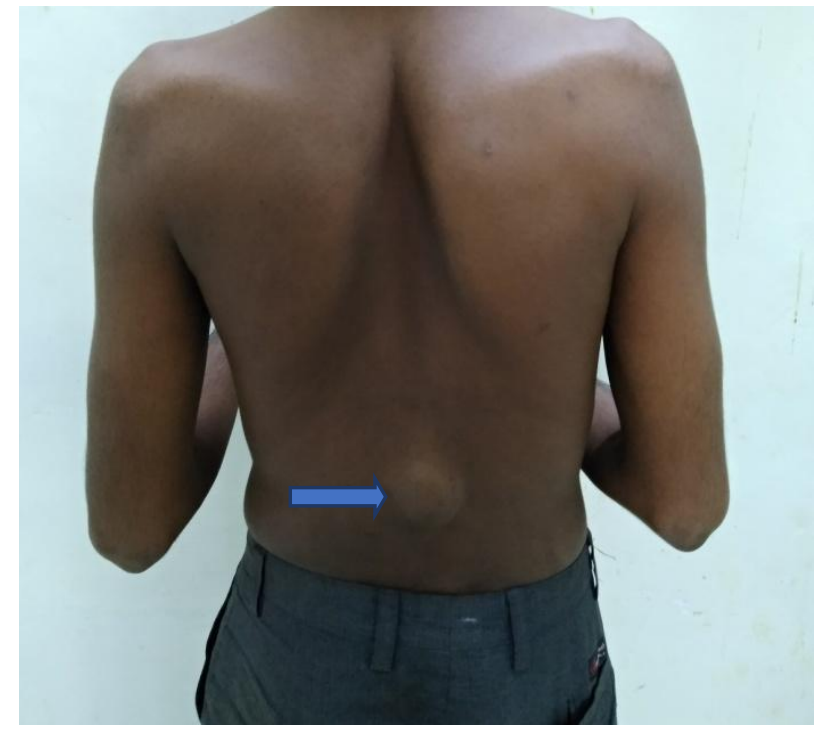

Fig No 7(A). photograph of pots spine showing bulge over lumbar spine(blue arrow).

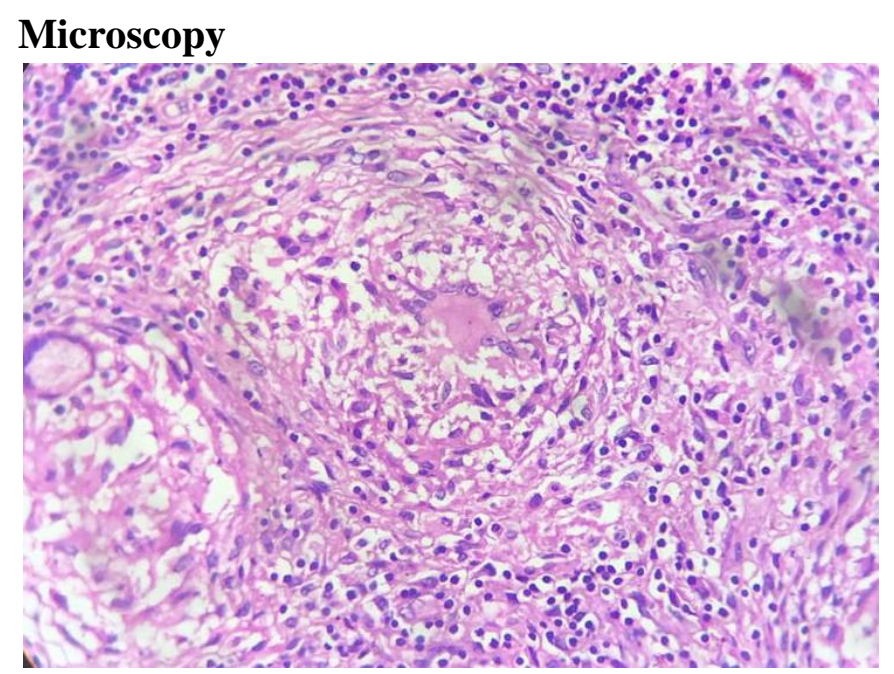

Fig No7 (B).(tuberculous osteomyelitis). Section study shows occasional granulomas composed of epithelioid cells and Langhans giant cells with surrounding lymphocytes.

\section{Discussion}

Table No 5: Comparison of Age wise distribution of bone lesions

\begin{tabular}{|l|c|c|c|}
\hline $\begin{array}{l}\text { Age } \\
\text { (years) }\end{array}$ & $\begin{array}{c}\text { V Manoja } \\
\text { et al }\end{array}$ & $\begin{array}{c}\text { Saadvi } \\
\text { Kethireddy et al }\end{array}$ & $\begin{array}{c}\text { Present } \\
\text { study }\end{array}$ \\
\hline$<20$ & 22 & 20 & 19 \\
\hline $21-40$ & 14 & 13 & 17 \\
\hline $41-60$ & 9 & 8 & 16 \\
\hline$>60$ & 5 & 5 & 8 \\
\hline Total & 50 & 48 & 60 \\
\hline
\end{tabular}

Bone lesions were more common in $<20$ years age group (31.66\%) which is similar with study done by V manoja et al (44\%), Saadvi Kethireddy et al $(41.66 \%)$.

Table No 6: Comparison of Sex distribution of bone lesions

\begin{tabular}{|l|c|c|c|}
\hline Auther & Male & Female & Total \\
\hline V Manoja et al & 31 & 19 & 50 \\
\hline Saadvi Kethireddy et al & 28 & 18 & 46 \\
\hline Modi D et al & 68 & 34 & 102 \\
\hline Present study & 36 & 24 & 60 \\
\hline
\end{tabular}

Males (36 out of 60 cases, $60 \%$ ) were affected more commonly than females (24 out of 60 cases, $40 \%$ ) with $\mathrm{M}: \mathrm{F}$ ratio as $1.5: 1$, which is similar with the study by $\mathrm{V}$ Manoja et al (M:F=1.6:1), Saadvi Kethireddy et al (M:F=1.6:1), Modi D et al $(\mathrm{M}: \mathrm{F}=2: 1)$.

Table No 7: Comparison of site distribution of bone lesions

\begin{tabular}{|l|c|c|c|}
\hline Location & $\begin{array}{c}\text { V Manoja } \\
\text { et al }\end{array}$ & $\begin{array}{c}\text { Saadvi } \\
\text { Kethireddy et al }\end{array}$ & $\begin{array}{c}\text { Present } \\
\text { study }\end{array}$ \\
\hline Femur & $27(54 \%)$ & $25(54.34 \%)$ & $20(33.33 \%)$ \\
\hline Tibia & $12(24 \%)$ & $11(23.9 \%)$ & $15(25 \%)$ \\
\hline Humerus & $7(14 \%)$ & $4(8.69 \%)$ & $11(18.33 \%)$ \\
\hline Radius & $2(4 \%)$ & $2(4.34 \%)$ & $7(11.66 \%)$ \\
\hline Ulna & $1(2 \%)$ & $2(4.34 \%)$ & $4(6.66 \%)$ \\
\hline Small bones & $1(2 \%)$ & $2(4.34 \%)$ & $3(5 \%)$ \\
\hline Total & $50(100 \%)$ & $46(100 \%)$ & $60(100 \%)$ \\
\hline
\end{tabular}

The most common site of bone lesion is femur (33.33\%), which is similar with the study by $\mathrm{V}$ Manoja et al and Saadvi Kethireddy et al which shows most common site is femur $(54 \%$ and $54.34 \%$ respectively.

Table No 8: Comparison of different nonneoplastic lesions of bone

\begin{tabular}{|c|c|c|}
\hline $\begin{array}{c}\text { Saadvi Kethireddy1 } \\
\text { et al }\end{array}$ & V Manoja et al & Present study \\
\hline osteomyelitis -7 & Osteomyelitis -6 & Osteomylitis- 16 \\
\hline $\begin{array}{c}\text { Tuberculous } \\
\text { osteomyelitis -4 }\end{array}$ & $\begin{array}{c}\text { Tuberculous } \\
\text { osteomyelitis-3 }\end{array}$ & $\begin{array}{c}\text { TB osteomyelitis - } \\
8\end{array}$ \\
\hline $\begin{array}{c}\text { Aneurysmal bone } \\
\text { cyst-3 }\end{array}$ & $\begin{array}{c}\text { aneurysmal bone } \\
\text { cyst - 2 }\end{array}$ & $\begin{array}{c}\text { Aneurismal bone } \\
\text { cyst- 5 }\end{array}$ \\
\hline $\begin{array}{c}\text { Fibrous dysplasia- } 2 \\
\text { Simple cyst- 1 }\end{array}$ & $\begin{array}{c}\text { Nonossifying } \\
\text { fibroma-1 }\end{array}$ \\
\hline $\begin{array}{c}\text { Non-ossifying } \\
\text { fibroma-1 }\end{array}$ & Fibrous dysplasia-1 & $\begin{array}{c}\text { Simple bone cyst- } \\
2\end{array}$ \\
\hline Total-18 & Total-13 & Total- 32 \\
\hline
\end{tabular}


In our study the most common non- neoplastic bone lesion is osteomyelitis (16/32), followed by tuberculous osteomyelitis (8/32), aneurysmal bone cyst (5/32), simple cyst (2/32), Non-ossifying fibroma (1/32).

Which is similar with study done by Saadvi Kethireddy1 et al the most common nonneoplastic bone lesion is osteomyelitis (7/18), followed by tuberculous osteomyelitis (4/18), aneurysmal bone cyst (3/18), fibrous dysplasia (2/18), simple cyst (1/13), Non-ossifying fibroma(1/18).

According to study done by V Manoja et al which shows the most common non- neoplastic bone lesion is osteomyelitis (6/13), followed by tuberculous osteomyelitis (3/13), aneurysmal bone cyst (2/13), simple cyst (1/13), fibrous dysplasia $(1 / 13)$.

Table No 9: Comparison of different benign tumors of bone

\begin{tabular}{|c|c|c|}
\hline $\begin{array}{c}\text { Saadvi Kethireddy1 } \\
\text { et al }\end{array}$ & V Manoja et al & Present study \\
\hline Giant cell tumor -12 & $\begin{array}{c}\text { Osteochondroma- } \\
22\end{array}$ & Osteochondroma- 8 \\
\hline Osteochondroma -6 & Osteoclastoma-10 & Giant cell tumor - 7 \\
\hline Osteoma- 3 & Osteoid osteoma- 1 & Osteoid osteoma- 4 \\
\hline Chondroblastoma-2 & Enchondroma- 1 & Chondroblastoma-2 \\
\hline Enchondroma- 2 & & Total-21 \\
\hline Total-25 & Total-34 & \\
\hline
\end{tabular}

According to our present study the most common benign neoplastic tumor is osteochondroma $(8 / 21)$ which is similar with the study done by $\mathrm{V}$ manoja et al which shows osteochondroma (22/34). Study by Saadvi Kethireddy1 et al shows most common benign tumor is giant cell tumor (12/25).

Table No 10: Comparison of different malignant tomors of bone

\begin{tabular}{|c|c|c|}
\hline $\begin{array}{c}\text { Saadvi Kethireddy1 } \\
\text { et al }\end{array}$ & V Manoja et al & Present study \\
\hline Osteosarcoma -2 & Osteosarcoma-1 & Osteosarcoma -2 \\
\hline Ewing's sarcoma- 1 & metastasis- 1 & Metastasis -2 \\
\hline & Ewing's sarcoma-1 & Ewing sarcoma-2 \\
\hline & & Chordoma-1 \\
\hline Total- 3 & Total- 3 & Total- 7 \\
\hline
\end{tabular}

Our present study shows osteosarcoma, ewing's sarcoma and mets ( 2 cases each) followed by chordoma which is similar with the study done by osteosarcoma (2/3) and osteosarcoma (1/3).

\section{Conclusion}

The most common lesions are non neoplastic followed by benign neoplastic tumors then malignant neoplastic tumors. Out of all the bone lesions osteomyelitis found to be most common. The most common site involved is femur. Bone lesions are more common in male than female. The histopathological examination is the gold standard to diagnose bone lesion when it is combined with relevant history and clinical data.

\section{Reference}

1. Rhutso Y, Laishram RS, Sharma LD, Debnath K. Histopathological evaluation of bone tumors in a tertiary care hospital in Manipur, India. J Med Soc 2013;27:1359.

2. Modi D, Rathod GB, Delwadia KN, Goswami HM. Histopathological study of bone lesions - A review of 102 cases. IAIM 2016;3:27-36. Back to cited text no.

3. Rafiq M, Tanwani AK. Spectrum of bone lesions at Pakistan institute of medical sciences. J Islamabad Med Dent Coll 2012;2:69-71.

4. Bonetumor.org, (internet), Massachusetts, Henry Degroot, Bone metastasis, Available from, Accessed on 20-11-2015.

5. Obalum DC, Giwa SO, Banjo AF, Akinsulire AT. Primary bone tumours in a tertiary hospital in Nigeria a 25 year review. Nigerian Journal of Clinical Practice. 2009 Jun;12(2):169-172.

6. Saadvi Kethireddy1, K. Raghu2, K. P. A. Chandra Sekhar3. Histopathological Evaluation of Neoplastic and NonNeoplastic Bone Tumours In A Teaching Hospital. J. Evolution Med. Dent. Sci./eISSN- 2278-4802, pISSN- 22784748/ Vol. 5/ Issue 86/ Oct. 27, 2016.

7. V Manoja, Divya Chevakula, K Suresh. Histopathological evaluation of bone 
lesions: A retrospective institutional study.

Med Pulse International Journal of Pathology. October 2019; 12(1): 01-04.

8. Dr. Shubhi Sharma, Dr. Nandita P. Mehta Histopathological Study of Bone Tumors, Ahmedabad, Gujarat, India, International Journal of Science and Research, Volume 4 Issue 12, December 2015.

9. F.B. Abdulkareem et al, Pathological study of Bone Tumors at National Orthopedic Hospital, Lagos Nigeria, West African Journal of Medicine.

10. Negash BE, Admasie D, Wamisho BL, Tinsay MW, bone Tumors at Addis Abbas University Ethiopia, Agreement Between Radiological and Histopathological Diagnosis - A 5 year analysis at Black Lion Teaching Hospital, Malawi Med J $1: 62-5$. 